\title{
Origin of photoluminescence signals obtained by picosecond-excitation correlation measurements
}

\author{
J. L. A. Chilla, O. Buccafusca, and J. J. Rocca \\ Center for Optoelectronic Computing Systems and Department of Electrical Engineering, \\ Colorado State University, Fort Collins, Colorado 80523
}

(Received 24 June 1993)

\begin{abstract}
We have developed a theory that explains the spectral distribution and the different time decay behaviors of the signals observed in picosecond excitation-correlation measurements of semiconductor materials. The results display good quantitative agreement with experiments on multiple quantum wells and show that band filling has an important role in determining the characteristics of the correlation signals. Two limit cases are found and mathematically characterized: the nondegenerate regime, where the signals are positive with exponential-like decays, and the degenerate regime, where band filling gives rise to negative signals. It is shown that the technique is a sensitive probe of the carrier dynamics not fully exploited in previous work.
\end{abstract}

\section{INTRODUCTION}

The time resolved photoluminescence (PL) technique known as population mixing or picosecond excitation correlation (PEC) spectroscopy was introduced in 1981 (Refs. 1 and 2 ) and since then it was widely employed to study carrier dynamics in semiconductor materials. ${ }^{1-10}$ This technique has the advantage of providing a time resolution limited by the laser pulse width and of requiring an experimental setup which is much simpler than that of other ultrafast methods of measuring the PL decay, such as frequency up conversion. ${ }^{11} \mathrm{~A}$ disadvantage arises from the fact that it is a correlation method, which requires careful interpretation of the measured signals.

In this technique two trains of laser pulses, usually with pulse widths in the picosecond or subpicosecond range, generate carriers which later recombine by radiative and nonradiative mechanisms. Due to the nonlinear relation between the luminescence and the carrier density, there is a correlation between the PL generated by both trains of pulses. By measuring the correlation of the PL as a function of the relative delay between the pulses it is possible to observe the dynamics of the photogenerated carriers.

According to this simple description, PEC signals can be expected to decay following the decay of the carrier population as the delay between the pulses is increased. These types of PEC signals, which are simple to interpret, have often appeared in the literature and the signal decay rate has been used to measure several fast processes in semiconductors such as carrier lifetimes, ${ }^{1,3}$ tunneling, ${ }^{4-6}$ minority carrier drift,${ }^{7}$ exciton formation time, ${ }^{6}$ and real space carrier transfer in $\mathrm{Al}_{x} \mathrm{Ga}_{1-x} \mathrm{As} / \mathrm{GaAs}$ heterostructures. ${ }^{8}$

However, the PL correlation signal can also change sign and slope depending on the excitation intensity and the wavelength at which the PL signal is detected. Perhaps due to its more difficult interpretation, this kind of signal has remained for the most part absent in the litera- ture. Some exceptions are Johnson and McGill, ${ }^{3}$ who attributed the negative signals to extremely long carrier lifetimes, Jørgensen and Hvam, ${ }^{9}$ who used the sign change in the PEC spectra of CdSe to identify the bound exciton PL peak, and Eakin and Ryan, ${ }^{10}$ who attributed the dip in the PEC signal to carrier cooling. However, to our knowledge such significant changes in the signal characteristics have not been thoroughly studied. Most of the previous work on the analysis of this technique is only qualitative $\mathrm{i}^{1,9,10}$ or has been restricted to spectrally integrated signals and low excitation intensities. ${ }^{3,6}$ The PEC technique merits detailed study because it is a sensitive probe ${ }^{6}$ of the dynamics of processes occurring in photoexcited materials that has not been fully exploited.

Herein we present a complete analysis of the spectrally resolved PEC signals that shows good agreement with measurements conducted on $\operatorname{In}_{x} \mathrm{Ga}_{1-x} \mathrm{P} / \mathrm{In}_{x} \mathrm{Al}_{1-x} \mathrm{P}$ multiple quantum wells (MQW's). As a result of our analysis, two distinct regimes (nondegenerate and degenerate) are found and characterized. When the excited carrier density is low the semiconductor is in a nondegenerate situation and the PEC signals present exponential-like decays that are easy to interpret. For high excited carrier density the semiconductor is degenerate and negative signals appear in the low energy zone of the spectrum, where the PL saturates. It was previously reported ${ }^{3}$ that the PEC signal is zero in absence of a nonradiative decay path. Our more complete analysis confirms the result for the nondegenerate regime, and shows that in the case of the degenerate regime only the spectrally integrated signal is equal to zero due to the compensation of positive and negative parts of the spectral distribution of the PEC signal. Furthermore, in the presence of a nonradiative decay path we find a quantitative relation between the ratio of negative and positive areas of the PEC signal and the rate of increase of the radiative efficiency with the excitation power.

In the next section we will present experimental results of PEC measurements showing unusual features such as 
changes in sign and non-exponential-like decays that motivated our study. Section III is devoted to the theoretical analysis of PEC signals. We first obtain analytical expressions for the two limiting cases of low (nondegenerate) and high (degenerate) carrier density, which provide a useful insight into the physics of the problem. Later we use numerical simulations to study the general case and obtain quantitative information on the physical parameters of the sample. In Sec. IV we discuss the interpretation of PEC signals and study their relation to the radiative branching ratio, and finally we summarize our results in Sec. V.

\section{PICOSECOND EXCITATION CORRELATION SIGNALS FROM In $\mathrm{Ga}_{1-x} \mathrm{P} / \mathrm{In}_{x} \mathrm{Al}_{1-x} \mathrm{P}$ MQW's}

We used PEC spectroscopy to perform time resolved $\mathrm{PL}$ measurements in $\operatorname{In}_{x} \mathrm{Ga}_{1-x} \mathrm{P} / \operatorname{In}_{x} \mathrm{Al}_{1-x} \mathrm{P} \mathrm{MQW}$ structures grown by gas source molecular-beam epitaxy. Our sample consists of a stack of $30 \operatorname{In}_{x} \mathrm{Ga}_{1-x} \mathrm{P}$ wells 8.5 $\mathrm{nm}$ thick separated by $22.5 \mathrm{~nm} \mathrm{In}_{x} \mathrm{Al}_{1-x} \mathrm{P}$ barriers as described in detail in Ref. 12. In an experimental setup similar to others reported in the literature, ${ }^{1,3,9}$ the sample, cooled to $10 \mathrm{~K}$ in a cryostat, is illuminated by two trains of synchronized laser pulses that are modulated separately at two different frequencies. The PL emitted by the sample is detected with a slow detector attached to a spectrometer.

The time integrated PL generated by either pump beam can be obtained by referencing the lock-in amplifier to the corresponding modulation frequency while the correlation between the PL generated by both pulses is obtained by referencing the lock-in amplifier to either the sum or difference of the chopper frequencies. A typical result of such measurements can be observed in Fig. 1 where we plot the time integrated PL (dashed line) and the PEC signal (solid line) as a function of the energy of the luminescence photons. These curves were obtained

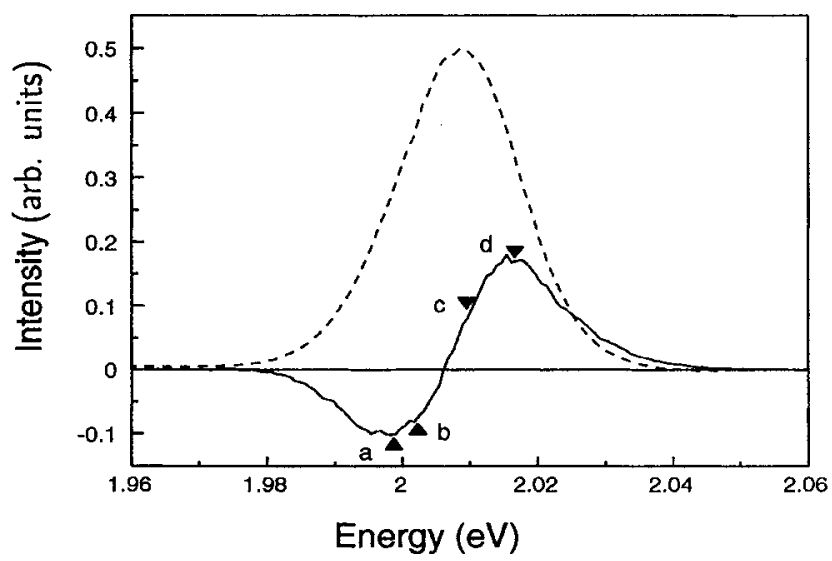

FIG. 1. Measured time integrated PL (dashed line) and PEC (solid line) spectra corresponding to $8 \mathrm{~mW}$ excitation power focusing with a $75 \mathrm{~mm}$ focal length lens. The energies corresponding to the decays of Fig. 2 are indicated. using a sync-pump dye laser with $8 \mathrm{~mW}$ of mean power in each beam and focusing onto the sample with a 75 $\mathrm{mm}$ focal length lens. The 2 ps pulses, with a central wavelength of $580 \mathrm{~nm}$, had a repetition rate of $76 \mathrm{MHz}$ and were modulated at $1.77 \mathrm{KHz}$ and $2.12 \mathrm{KHz}$.

The time integrated PL in Fig. 1 is almost symmetric in energy with a shape very similar to a Gaussian curve. As the luminescence comes from many quantum wells with fluctuations in width of around $5 \%,{ }^{12}$ at low temperatures the line shape is expected to be Gaussian. ${ }^{13}$ With measurements of the PL at low excitation intensity we find this broadening to be $8 \mathrm{meV}$. This effect will be taken into account when comparing to the predictions of the theory for PEC signals.

To obtain the corresponding PEC signal shown in Fig. 1 both trains of pulses were adjusted to overlap temporally and spatially on the sample. In this condition we observe the $\mathrm{PEC}$ signal in the entire energy range spanned by the $\mathrm{PL}$, but as shown in the figure the line shape is very different from that of the $P L$, with positive $P E C$ signals in the high energy side and negative signals in the low energy side.

The temporal dependence of the cross correlation signal is obtained by delaying in time one train of pulses with respect to the other. By means of the spectrometer one can choose the energy of the detected photons and obtain the decay of the PEC signal for different energies. Figure 2 shows the PEC signal as a function of the relative delay between the trains of pulses for several zones in the spectrum that are labeled by the markers in Fig. 1. These curves were taken using a manual translation stage with one data point each 33 ps.

As Fig. 2 clearly shows, one can obtain very different PEC decay signals depending on the sampled energy region of the spectrum. Curves (a) and (d) are examples of decays corresponding to the low and high energy ends of the spectrum, while curves (b) and (c) illustrate the observed continuous change in the shape of the decays with energy. At high energy (d) we obtain an exponential-like decay. At lower energy (c) the signal first increases and then decays. As the correlation signal is symmetric, this initial increase can be described as a dip appearing at short delays. This dip grows further if we continue to-

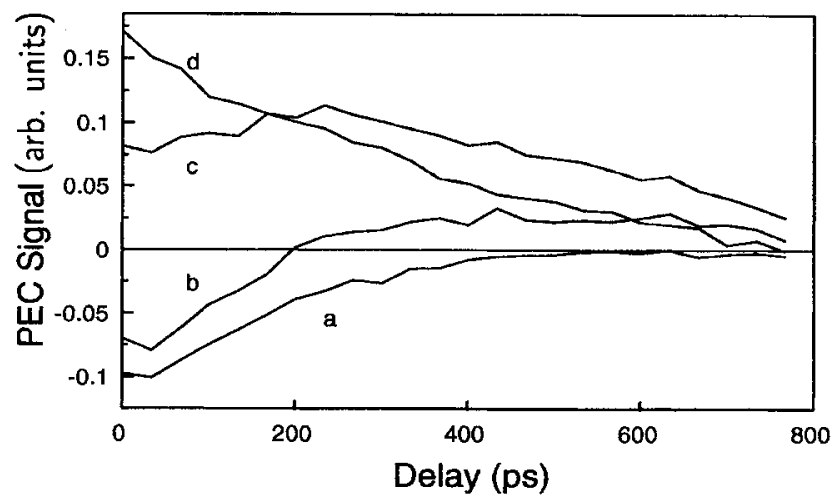

FIG. 2. PEC signal decays measured at the energies labeled by the markers in Fig. 1. The lines connect experimental points separated by $33 \mathrm{ps}$ in delay. 
wards low energy (b) and finally the signal becomes a negative exponential-like decay at the low energy end of the spectrum (a). Such variety of shapes from a single experiment clearly shows that the PEC signal is more complicated than a simple sampling of the decay of the photoexcited carrier population. Consequently, a more detailed analysis is needed if the lifetimes and other parameters are to be extracted from these data.

In order to understand the origin of this behavior we performed PEC measurements changing the power of the pump pulses. PEC spectra at zero delay corresponding to different pump powers, with PEC intensities normalized for ease of comparison, are shown in Fig. 3. The spectrum taken at low power $(0.4 \mathrm{~mW})$ is completely positive and its shape is very similar to the corresponding time integrated PL (not plotted). When we increase the power of the pump beams the low energy side of the spectrum first becomes negative, and then the area of the negative part increases as shown in Fig. 3 for excitation powers of $3.8 \mathrm{~mW}$ and $8.7 \mathrm{~mW}$. Series of measurements done at different powers show that the increase in the negative area with power saturates when negative and positive areas are approximately equal. The origin of these PEC signals is explained by the theoretical analysis presented in the next section.

\section{THEORETICAL ANALYSIS}

As it has been previously recognized, ${ }^{6,9}$ the PL correlation signal obtained by means of this technique is a measure of the nonlinearity of the detected PL with the excitation power. More precisely, the PEC signal is the difference between the PL generated by the two delayed pulses and twice that generated by only one excitation pulse. ${ }^{6}$ To show this we will consider the situation depicted in Fig. 4. Figure 4(a) represents the electrical input to the lock-in amplifier obtained by blocking one of the pump beams, which can be expressed as

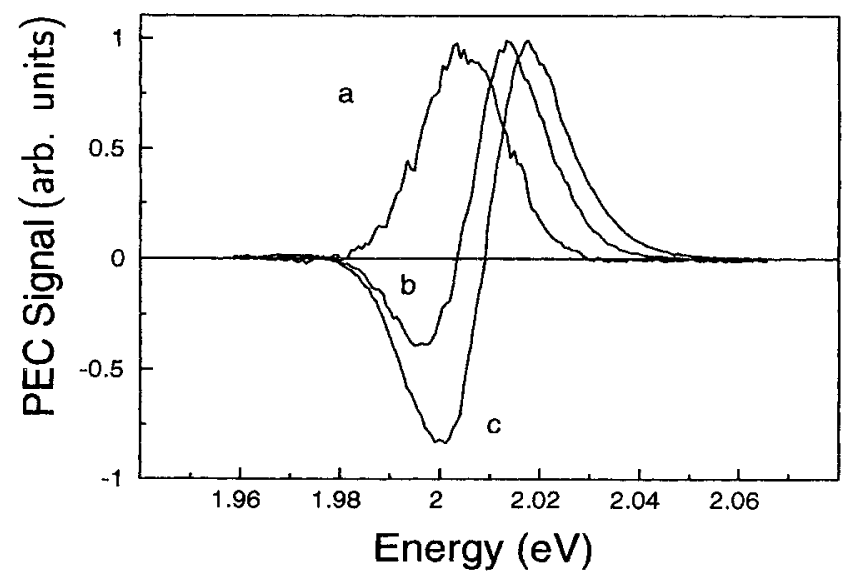

FIG. 3. PEC spectra measured at different excitation powers (a) $0.4 \mathrm{~mW}$, (b) $3.8 \mathrm{~mW}$, and (c) $8.7 \mathrm{~mW}$. Curves are normalized for ease of comparison.

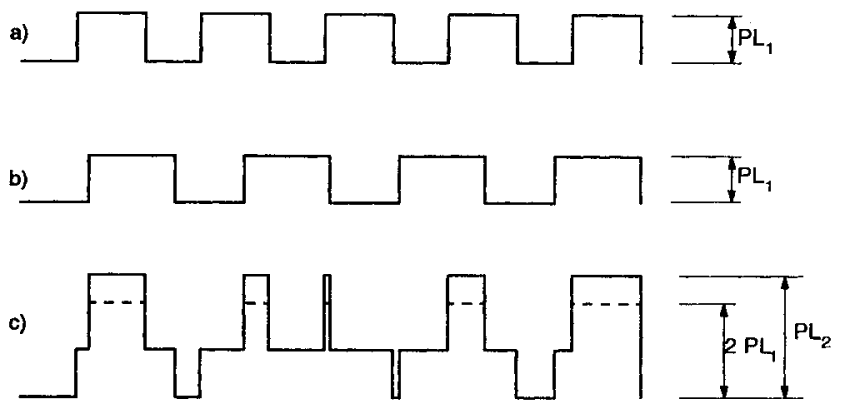

FIG. 4. Electrical signals entering the lock-in. Traces (a) and (b) represent the signal obtained when blocking one pump beam or the other. Trace (c) is obtained when both beams are present.

$$
S_{a}(t)=P_{L 1} c\left(\omega_{a} t\right)
$$

Due to its slow response time compared to the PL duration, the detector produces an electrical signal $P_{L 1}$, proportional to the time integral of the detected PL. As the pump beam is chopped at a frequency $\omega_{a}$ slower than the response of the detector, the electrical signal is affected by the transmission function of the chopper $c\left(\omega_{a} t\right)$.

A similar signal is obtained by blocking only the other pump beam, as shown in Fig. 4(b). When the intensity of both pump beams is the same, the only difference between the two signals is due to the chopper frequency, i.e.,

$$
S_{b}(t)=P_{L 1} c\left(\omega_{b} t\right) .
$$

If the emitted PL were linear, the electrical signal with both pump beams unblocked would be simply the sum of the signal coming from each pump, as shown by the dotted line in Fig. 4(c). However, due to the nonlinearity of the process, when both pump beams are present, the detector produces a signal $P_{L 2}$, different from $2 \times P_{L 1}$ as shown by the solid line in Fig. 4(c). The signal can be expressed as the linear term plus a nonlinear correction that appears only when both beams are present,

$S_{a b}=P_{L 1}\left[c\left(\omega_{a} t\right)+c\left(\omega_{b} t\right)\right]+\left(P_{L 2}-2 P_{L 1}\right) c\left(\omega_{a} t\right) c\left(\omega_{b} t\right)$.

By simple inspection of Eq. (3) we note that the only components at frequencies $\left(\omega_{a}+\omega_{b}\right)$ and $\left(\omega_{a}-\omega_{b}\right)$ arise from the nonlinear term and are proportional to $P_{L 2}$ $2 \times P_{L 1}$. Therefore the output from the lock-in as a function of the energy $\epsilon$ and delay $\delta$ can be expressed as

$$
s(\epsilon, \delta)=P_{L 2}(\epsilon, \delta)-2 P_{L 1}(\epsilon),
$$

where $P_{L 2}(\epsilon, \delta)$ is the time integrated photoluminescence at energy $\epsilon$ generated by two pulses separated by a delay $\delta$, while $P_{L_{1}}(\epsilon)$ is the PL originating from only one pulse. It is clear from (4) that the PEC signal vanishes when the dependence of the PL with excitation power is linear.

In order to obtain the PEC signal as a function of the detection wavelength and relative delay between the pulses, the emitted luminescence in the conditions of the 
experiment must be computed. For that purpose we built a simplified theoretical model of the processes that take place in the semiconductor after optical excitation. Typical systems use pulses with durations in the order of a few picoseconds or less that are absorbed in the sample by generating carriers. Shortly after generation the energy distribution of the carriers is thermal, characterized by quasi-Fermi-levels for electrons and holes and a carrier temperature $T_{0}$, different, in principle, from the lattice temperature $T_{L} \cdot{ }^{14}$ The processes of generation and carrier-carrier thermalization coexist and last a few picoseconds, while recombination time is usually several hundred picoseconds, thus the great majority of the photons will be emitted when the carrier distribution is thermal. As the PEC signals are computed from the time integrated PL we can safely neglect the photons emitted from nonthermal distributions and assume that upon arrival of the laser pulse a certain population of photoexcited carriers is instantaneously generated at temperature $T_{0}$. The temperature of the carriers later decays to that of the lattice in a longer time scale. ${ }^{14}$

According to Ref. 15, the number of photons emitted in the form of luminescence in an interval $d \epsilon$ around the energy $\epsilon$ during the time interval $d t$ is

$$
d P_{L}(\epsilon, t)=B n\left(\frac{m_{h} \epsilon}{m_{h}+m_{e}}\right) p\left(\frac{m_{e} \epsilon}{m_{h}+m_{e}}\right) d \epsilon d t .
$$

In this expression $B$ is the radiative recombination rate constant, $\epsilon=E-E_{1}$ measures the energy of the photon relative to the energy of a transition originated at the bottom of the band $\left(E_{1}\right), m_{e}$ and $m_{h}$ are the effective masses of electrons and holes, while $n(\epsilon)$ and $p(\epsilon)$ are the densities of electrons and holes as a function of the energy measured from the bottom of each band. Expression (5) takes into account the conservation of the wave number $k$ required in photon emission and assumes parabolic bands. Integration of $n(\epsilon)$ and $p(\epsilon)$ over energy gives the total density of photoexcited electrons $n$ and holes $p$.

If we consider that the population of carriers is affected by both radiative recombination and some additional nonradiative mechanism we can write the differential equation giving the temporal evolution of the carrier population, where we have included the customary generation term $G(t)$.

$$
\begin{aligned}
\frac{d n}{d t}= & G(t)-\frac{n}{\tau_{\mathrm{nr}}} \\
& -\int d \epsilon B n\left(\frac{m_{h} \epsilon}{m_{h}+m_{e}}\right) p\left(\frac{m_{e} \epsilon}{m_{h}+m_{e}}\right) .
\end{aligned}
$$

To obtain the time integrated PL signals as appearing in expression (4), Eq. (5) should be temporally integrated after insertion of the evolution of the carrier population determined by the differential equation in (6). We first consider two limiting cases of very low (nondegenerate) and high (degenerate) carrier concentration in which the further simplification of constant carrier temperature allows an analytical solution to be found. Study of these particular situations provides a very useful insight into the problem, while the general case of arbitrary carrier concentration and cooling rate is later addressed in Sec. III C with numerical simulations.

\section{A. Nondegenerate regime}

When the photoexcited carrier density is low, the quasi-Fermi-levels are located out of the bands and both $n(\epsilon)$ and $p(\epsilon)$ are the exponential tails of the Fermi distribution. The conditions of nondegeneracy for a MQW are

$$
\begin{aligned}
& n<g_{e} k T \ln (2), \\
& p<g_{h} k T \ln (2),
\end{aligned}
$$

with

$$
\begin{aligned}
& g_{e}=\frac{m_{e}}{(\pi \hbar)^{2}}, \\
& g_{h}=\frac{m_{h}}{(\pi \hbar)^{2}},
\end{aligned}
$$

where $g_{e}$ and $g_{h}$ are the two-dimensional densities of states for electrons and holes, $k$ is the Boltzmann constant, and $T$ is the absolute temperature of the carriers which is assumed constant. It is evident from these expressions that at low temperature, even relatively low carrier densities can lead to a degenerate situation.

By performing the integral of (6) in the nondegenerate situation we obtain a term proportional to the product of the populations of holes and electrons.

$$
\frac{d n}{d t}=G(t)-\frac{n}{\tau_{\mathrm{nr}}}-B\left(\frac{1}{k T}\right)^{2} e^{-\epsilon / k T} n p .
$$

This situation is implicitly assumed throughout the literature for the analysis of PEC signals and corresponds to the case analyzed by Johnson and McGill. ${ }^{3}$ As concluded there, a purely radiative recombination does not give rise to a correlation signal. The measured PEC signal is originated from the nonlinearity introduced in the PL by the existence of a nonradiative decay path.

If we consider that the decay is dominated by the nonradiative mechanism, Eq. (10) can be approximately solved with exponential decays

$$
\begin{aligned}
& n(t)=n_{0} e^{-t / \tau_{e}} \\
& p(t)=n_{0} e^{-t / \tau_{h}} .
\end{aligned}
$$

In these expressions $\tau_{e}$ and $\tau_{h}$ are the lifetimes of electrons and holes, and $n_{0}$ is the initial density of photoexcited carriers.

By replacing (11) and the exponential energy distribution in (5), and performing the necessary integrations, we obtain the correlation signal as a function of the photon energy $\epsilon$ and the delay $\delta$.

$$
s(\epsilon, \delta)=B\left(\frac{n_{0}}{k T}\right)^{2} \frac{\tau_{e} \tau_{h}}{\tau_{e}+\tau_{h}} e^{-\epsilon / k T}\left(e^{-\delta / \tau_{e}}+e^{-\delta / \tau_{h}}\right) .
$$


As expected, this signal is fairly simple to interpret; its decay rate is the same at all wavelengths and equal to the decay rate of the carrier population. The PEC spectrum in this case turns out to be simply proportional to the time integrated PL spectrum.

\section{B. Degenerate regime}

In this case the quasi-Fermi-levels of electrons and holes are located within the corresponding band. To allow for a simple mathematical treatment we will assume the temperature $T=0$, so that both $n(\epsilon)$ and $p(\epsilon)$ take the value of the density of states below the Fermi level and a value of zero for energies above that level. The more general case of a higher temperature is discussed in Sec. III C.

For $T=0$, the integrand in (6) is different from zero only when both $n(\epsilon)$ and $p(\epsilon)$ are not zero, which occurs only for energies below the lowest of the quasi-Fermilevels for electrons and holes as corrected by the relation of masses in (5). As the Fermi level is determined by the population, the result of the integral in (6) will be proportional to the population of either electrons or holes depending on which one decays the fastest. In what follows we will assume the decay for electrons to be faster than for holes; in the opposite situation the calculations are analogous.

$$
\frac{d n}{d t}=G(t)-n\left(\frac{1}{\tau_{\mathrm{nr}}}+B\left(g_{e}+g_{h}\right)\right)
$$

This equation is readily integrated and it yields an exponential decay of the electron population. The lifetime has two components: nonradiative $\tau_{\mathrm{nr}}$ and radiative $\tau_{r}=1 / B\left(g_{e}+g_{h}\right)$.

Once the time evolution of the carrier population is determined, expression (5) can be used to compute the time integrated PL as a function of the excess energy $\epsilon$,

$$
P_{L}(\epsilon)= \begin{cases}B g_{e} g_{h} \tau \ln \left(\frac{n}{g \epsilon}\right) & , 0<\epsilon<n / g \\ 0 & , \epsilon>n / g\end{cases}
$$

where

$$
\begin{aligned}
& \frac{1}{\tau}=\frac{1}{\tau_{\mathrm{nr}}}+\frac{1}{\tau_{r}}, \\
& \frac{1}{g}=\frac{1}{g_{e}}+\frac{1}{g_{h}} .
\end{aligned}
$$

Expression (14) was obtained assuming excitation with a single pulse that generates an initial density of carriers $n$.

We next proceed to define a set of parameters $\left(n_{0}\right.$, $\tau_{1}, \alpha$, and $\beta$ ) that fully characterizes the photoluminescence emitted when two delayed pulses are incident on the sample. If only one of the pulses hits the sample, it will generate an initial carrier density $n_{0}$ that will decay with a lifetime $\tau_{1}$. The second pulse will encounter a partially saturated absorption and will thus generate a lower carrier density $n=\alpha(\delta) n_{0}$. The parameter $\alpha(\delta)$ accounts for absorption saturation effects and depends on the incoming power and the time $\delta$ elapsed since the arrival of the first pulse. This parameter is included for completeness of the model, although $\alpha$ is often close to 1 in many practical situations.

We will define another parameter $\beta$ from the relation $\eta_{2}=\beta(\delta) \eta_{1}$, where $\eta_{1}$ denotes the radiative branching ratio, i.e., the fraction of carriers that recombine through the radiative path when only one of the pulses is present and $\eta_{2}$ is the radiative branching ratio when both pulses illuminate the sample. We will later use this definition to establish a mathematical expression for the previously recognized connection ${ }^{3}$ between $\mathrm{PEC}$ signals and radiative efficiency.

In the particular case of $T=0$ it can be shown that $\tau_{2}=\beta(\delta) \tau_{1}$, where $\tau_{2}$ is the lifetime of the carriers generated by the second pulse. Expression (13) shows that for $T=0$ the radiative decay time does not depend on the carrier density, thus in that case $\beta$ will account only for a saturation of the nonradiative decay path.

With the previous definitions and replacing the adequately modified equation (14) into (4) we obtain an expression that describes the spectral and delay dependence of the PEC signal,

$$
s(\epsilon, \delta)= \begin{cases}0, & \epsilon \geq \frac{n_{0}}{g}\left[e^{-\delta / \tau_{1}}+\alpha(\delta)\right] \\ s_{3}, & \frac{n_{0}}{\boldsymbol{g}} \leq \epsilon<\frac{n_{0}}{g}\left[e^{-\delta / \tau_{1}}+\alpha(\delta)\right] \\ s_{2}, & \frac{n_{0}}{\boldsymbol{g}} e^{-\delta / \tau_{1}} \leq \epsilon<\frac{n_{0}}{\mathbf{g}} \\ s_{1}, & 0 \leq \epsilon<\frac{n_{0}}{g} e^{-\delta / \tau_{1}}\end{cases}
$$

with

$$
\begin{gathered}
s_{1}=B g_{e} g_{h}\left[\delta+\tau_{1} \beta(\delta) f(\epsilon, \delta)-2 \tau_{1} \ln \left(\frac{n_{0}}{g \epsilon}\right)\right] \\
s_{2}=B g_{e} g_{h} \tau_{1}\left[\beta(\delta) f(\epsilon, \delta)-\ln \left(\frac{n_{0}}{g \epsilon}\right)\right] \\
s_{3}=B g_{e} g_{h} \tau_{1} \beta(\delta) f(\epsilon, \delta) \\
f(\epsilon, \delta)=\ln \left(\frac{n_{0}\left[e^{-\delta / \tau_{1}}+\alpha(\delta)\right]}{g \epsilon}\right)
\end{gathered}
$$

Typical results are plotted in Fig. 5 which shows the PEC spectrum for zero delay and the dependence of the PEC signal with the delay $\delta$ for different energies $\epsilon$. The dashed line in Fig. 5 is the result of expressions (17)(21), while the solid curves are obtained after Gaussian broadening of the dashed curves to take into account the spectral broadening caused in a MQW sample by the well-to-well fluctuations in width. ${ }^{13}$ These curves are in qualitative agreement with the experimental data in Figs. 1 and 2 showing that this simple model includes the physics underlying the wide variety of observed PEC signals. A quantitative comparison of the calculated and measured PEC signals is included in the next section, which treats the case of arbitrary photoexcited carrier density and $T>0$. 


\section{General case}

For arbitrary photoexcited carrier density and $T>0$, the differential equation (6) is solved numerically. We will assume that the carrier temperature decays exponentially from the initial value to the lattice temperature with a time constant $\tau_{T}$.

$$
T(t)=T_{L}+\left(T_{0}-T_{L}\right) e^{-t / \tau_{T}} .
$$

Once the carrier density and temperature are known, the quasi-Fermi-level for electrons (holes) can be computed from requiring the integral of the Fermi distributions to be equal at all times to the total electron (hole) density. A FORTRAN program was developed that computes the temporal dependence of the PL according to (5) and performs the necessary numerical integrations to evaluate the PEC signal [expression (4)]. This program was used to fit the experimental data presented in Sec. II. The results of the theoretical predictions are shown in Figs. 6 and 7.

Figure 6 shows the theoretical PEC decay signals corresponding to the same energies as the experimental ones plotted in Fig. 2. A notably good agreement with the
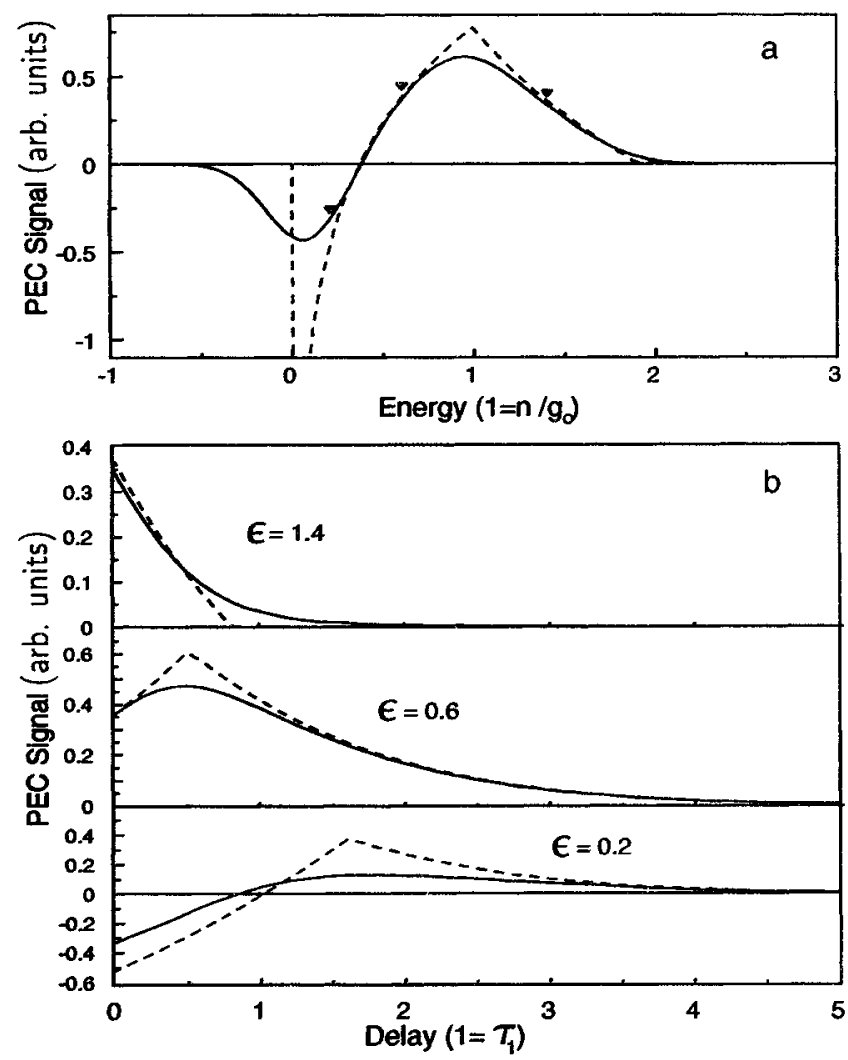

FIG. 5. PEC signals computed for $T=0$. Analytical expressions (17)-(21) are plotted in dashed lines. Solid lines show the result of Gaussian broadening. (a) PEC spectrum. The energy is measured in units of the initial Fermi level for one pulse. (b) Decay plots at the energies $(\epsilon)$ indicated. The delay is measured in units of the lifetime.

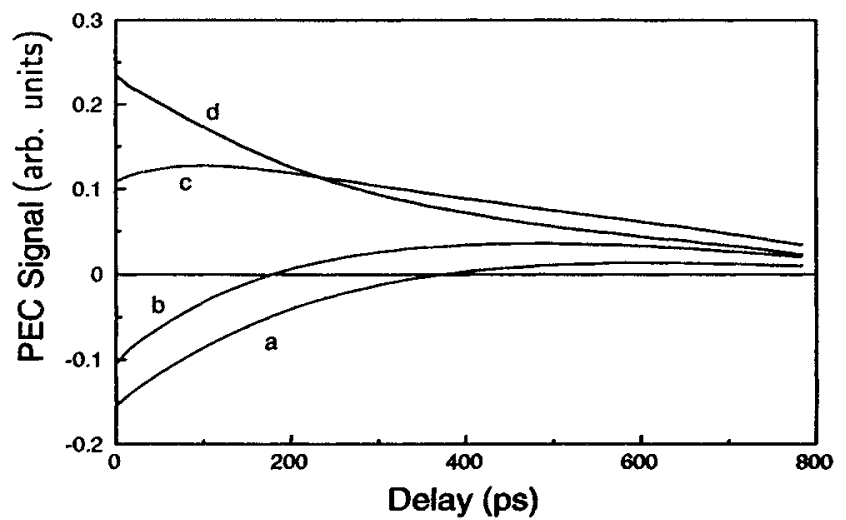

FIG. 6. Simulated PEC signal decays corresponding to the same energies as the experiment of Fig. 2. The curves were computed using parameters obtained by simultaneous fitting of the PEC spectra at different delays.

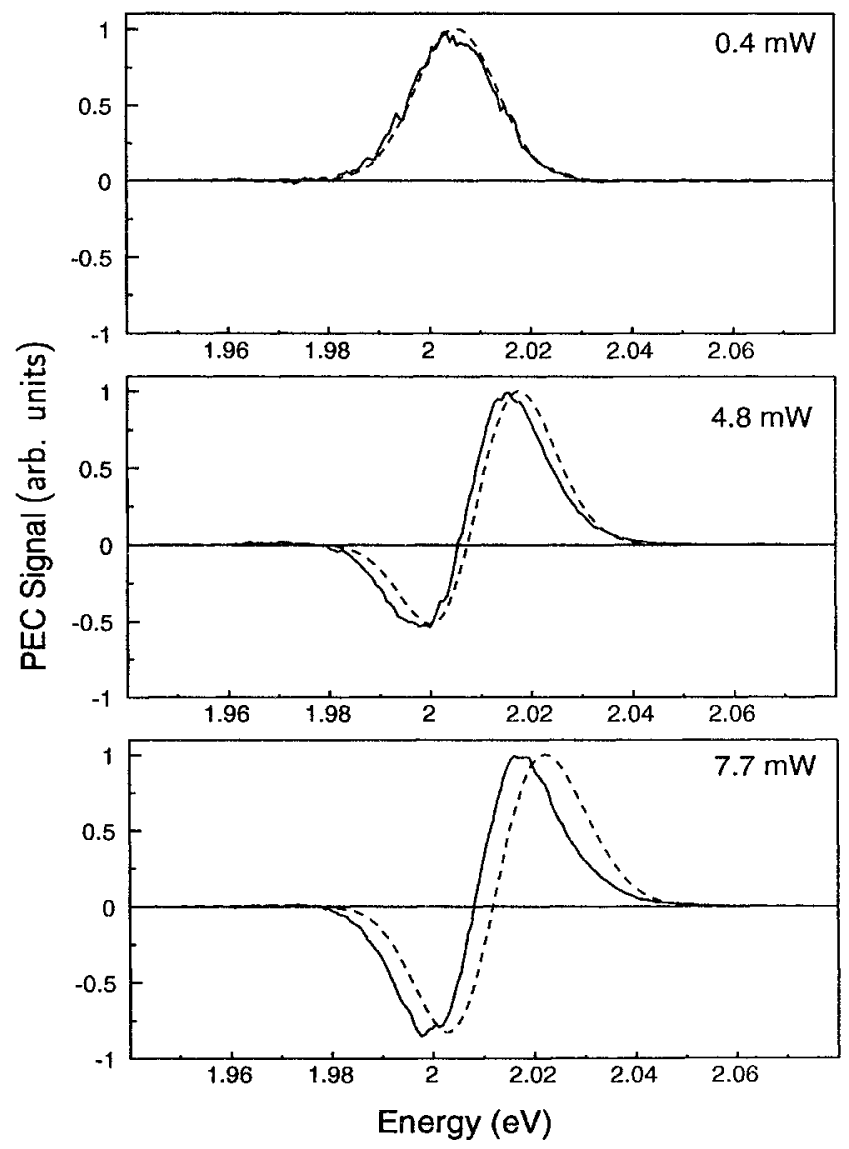

FIG. 7. Computed PEC spectra at different excitation powers (dashed lines) compared with the corresponding experimental spectra (solid lines). The energy shift observed at high power is due to band-gap renormalization effect, which is not accounted for in our model. 
experiment was obtained without the need to include carrier cooling effects, using material constants from the literature ${ }^{12}$ and only three fitting parameters that affect different features of the signal: the carrier density, the nonradiative decay time, and the bimolecular radiative recombination rate constant. The relation between radiative and nonradiative rates affects the shape of the spectra while their magnitude acts on the duration of the decays and the carrier density affects the shape and width of the PEC spectra. Making a simultaneous fitting of PEC spectra taken at different delays we obtained the following values for the parameters: $n=1.8 \times 10^{11} \mathrm{~cm}^{-2}$ when pumping with $8 \mathrm{~mW}$ per pump beam, $\tau_{\mathrm{nr}}=800$ ps, and $B=4.4 \times 10^{-17} \mathrm{~cm}^{2} \mathrm{eV} / \mathrm{ps}$. According to these values we inject a carrier density of $2.25 \times 10^{10} \mathrm{~cm}^{-2}$ per $\mathrm{mW}$ of pump power, in agreement with estimates from the intensity of the excitation and sample absorption. With the parameters from our fitting the radiative lifetime result is $300 \mathrm{ps}$, giving a total lifetime of 220 ps. To verify the accuracy of the obtained lifetimes we performed independent measurements on the same sample using a synchro-scan streak camera. These measurements yielded a (not perfectly exponential) decay with a lifetime of around $210 \mathrm{ps}$. It should be stressed that while the streak camera measurement gives almost the same total lifetime, the PEC technique gives more information with increased temporal resolution, even in the degenerate regime where simple exponential fittings are not possible.

To further test the theory we used the parameters obtained in the fitting to simulate measurements at different pump powers and compared the result with the experiment obtaining the very good agreement shown in Fig. 7. The figure shows the PEC spectra measured at three different powers and the corresponding predictions of the model. At low carrier density the theory and experiment match perfectly, while for higher carrier density the theory accurately predicts the change in shape of the signal but the experimental curve is slightly displaced to lower energy. The sign and magnitude of the displacement is consistent with band gap renormalization ${ }^{16}$ due to the high carrier concentration effect, which is not accounted for in our simple model.

\section{INTERPRETATION OF PEC SIGNALS}

The analysis of the PEC technique shows that band filling effects account for many of the previously observed changes in the signal with pump power and detected photon energy. Previous PEC measurements performed on $\mathrm{GaAs} / \mathrm{Ga}_{1-x} \mathrm{Al}_{x} \mathrm{As}$ quantum wells have shown PEC decay signals that are exponential-like decays at high energy in the PEC spectrum, and which at low energy display an initial increase that was attributed to carrier cooling effects. ${ }^{10}$ To justify the assumption of carrier cooling, Ref. 10 included calculations assuming a nondegenerate situation, where the product $n(\epsilon, t) \times p(\epsilon, t)$ is proportional to the PL intensity. The time dependence of this population product was computed and found to have a behavior similar to that of the measured PEC signals when the cooling time $\tau_{T}$ was of the order of the lifetime $\tau_{c}$ of the carriers.

However, calculations resulting from the model described in the previous section show the initial increase in the PEC signal is not due to carrier cooling as previously suggested. Figure 8 shows the numerically computed PL intensity and the PEC signal for different carrier cooling times with the detection energy set at the bottom of the band and assuming a nondegenerate situation. The top curves with instantaneous carrier cooling reproduce the analytical result of expression (12), i.e., the PEC signal decays following the carrier population decay while the $\mathrm{PL}$ intensity decays at twice that rate. The other curves show the effect of carrier cooling in a nondegenerate situation: the PL intensity corresponding to an energy at the bottom of the band initially increases because the rearrangement in energy of the carriers due to carrier cooling overcomes the decrease in total carrier population. However, the PEC signal does not show an initial increase, meaning that carrier cooling cannot explain the dips in
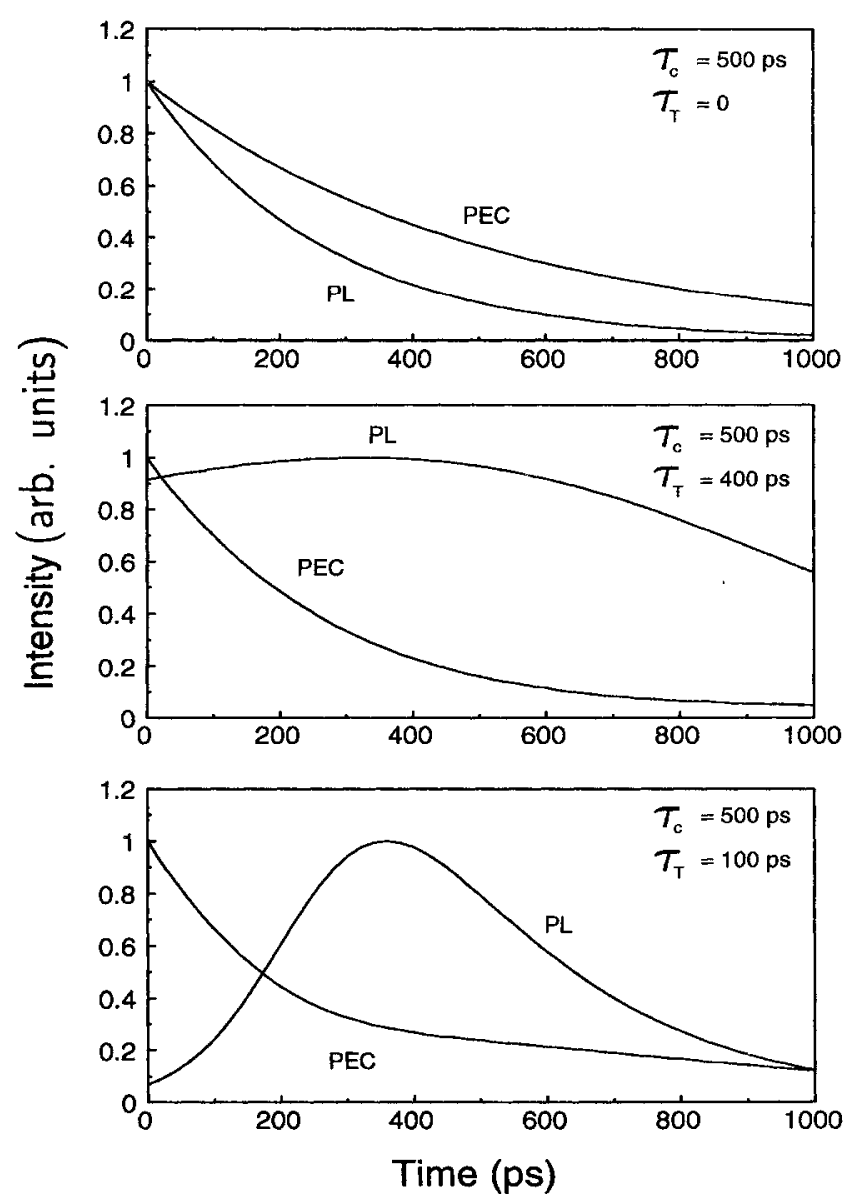

FIG. 8. Computed PL as a function of time and PEC signal as a function of delay with detected photon energy at the bottom of the band, for different values of lifetime $\left(\tau_{c}\right)$ and cooling time $\left(\tau_{T}\right)$. Temperature and initial carrier density correspond to the nondegenerate regime. Carrier cooling is observed to produce dips in the time dependent PL but not in the PEC signal. 
the PEC signals in a degenerate situation even when it produces dips in the time dependence of the PL intensity.

We instead attribute the initial increase of the PEC signals to band filling. If the carrier density is close to the condition of degeneracy [Eq. (7)] in the transition between the two regimes previously discussed, band filling is not strong enough to produce negative signals but manifests itself as the observed dips in the signals.

As the PEC signal changes shape affected by the change in carrier dynamics (Fig. 8), the technique could still be used to study carrier cooling by carefully fitting a set of spectrally resolved measurements with the predictions of the theoretical model of Sec. IIIC.

Another interesting result from the analysis discussed in Sec. III is a deeper understanding of the previously established connection ${ }^{3}$ between PEC signals and radiative efficiency. The spectrally and time integrated PL can be expressed as a product of the initial carrier density times the radiative branching ratio $\eta$ defined as the number of electrons that recombine radiatively relative to the total number of carriers

$$
P_{L}=\eta n_{0}
$$

We can use this relation to obtain the spectrally integrated correlation signal

$$
s=2 n_{0}\left(\eta_{2}-\eta_{1}\right)=2 n_{0} \eta_{1}(\beta-1) .
$$

Here we used the parameter $\beta(\delta)=\eta_{2} / \eta_{1}$ which measures the increase in radiative efficiency with excitation power. To compute the area of the negative part of the PEC spectrum we use expressions (17)-(21). The relation to be obtained by using those results is valid for $T=0 \mathrm{~K}$, but it is not expected to be affected dramatically by the carrier temperature because the negative part of the PEC spectrum originates in the zones of the band that are completely filled with carriers and thus in that energy zone the distribution is the same, independent of the carrier temperature. The negative area for $T=0$ is

$$
s_{\ldots}=n_{0} \eta_{1}(2-\beta) 2^{\beta /(\beta-2)} .
$$

From these relations simple algebra gives

$$
\begin{gathered}
s_{+}=s+s_{-}=n_{0} \eta_{1}\left[(2-\beta) 2^{\beta /(\beta-2)}+2(\beta-1)\right], \\
\frac{s_{-}}{s_{+}}=\frac{(\beta-2) 2^{\beta /(\beta-2)}}{(\beta-2) 2^{\beta /(\beta-2)}-2(\beta-1)} .
\end{gathered}
$$

Expression (27) shows that the ratio of areas is determined by the way the branching ratio changes with excitation power. The radiative rate constant depends on the overlap of the electron and hole distributions in $k$ space, and increases with carrier density while the semiconductor is nondegenerate and then saturates at the value $B\left(g_{e}+g_{h}\right)$. In the presence of a parallel nonradiative decay process, the radiative efficiency initially in- creases with excitation power and then saturates at a certain value. This means the parameter $\beta$ takes values larger than unity for low carrier density, and approaches 1 as the density increases. With this behavior of $\beta$ expression (27) (solid line in Fig. 9) predicts the increase of negative area with excitation power that can be observed in Fig. 3. If the decay is purely radiative, however, $\beta=1$ for all carrier densities corresponding to equal positive and negative areas. This can only be observed at high carrier densities because in the nondegenerate regime the PEC signal corresponding to this situation is zero.

Numerical simulations in a variety of conditions were performed in order to confirm the validity of Eq. (27) in the general case. Figure 9 shows a plot of Eq. (27) along with points obtained from simulations in which the parameters (excitation power, temperature, cooling rate, lifetimes) were chosen at random in a range spanning at least one order of magnitude, which correspond to values of $\eta$ from $3 \times 10^{-4}$ to 0.3 . This large variation in the parameters produced the comparatively small dispersion in the points that can be observed in the figure. As expected, the simulations are closer to the result of Eq. (27) when the ratio of areas approaches unity, i.e., when the carrier density is high and the distribution is similar to that of $T=0$.

\section{SUMMARY}

Although many studies have been previously conducted using the PEC technique, the lack of a detailed analysis implicitly restricted its applicability to the nondegenerate regime. Even in that case, as the PEC signal is a correlation measurement, the interpretation of experimental results is not always straightforward and the use of only qualitative reasoning in the analysis of the signals can lead to erroneous interpretations.

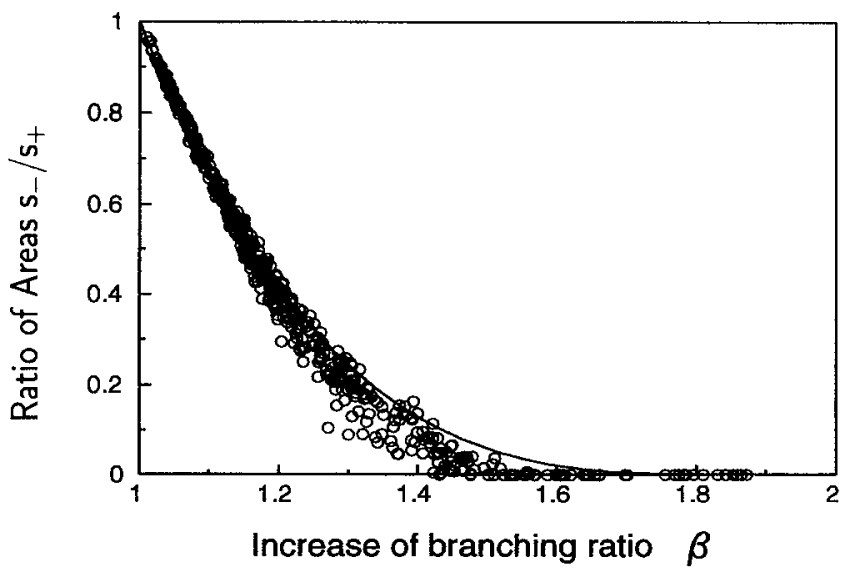

FIG. 9. Relation between the ratio of areas and the increase of radiative branching ratio, $\beta$. The solid line is the analytical relation computed at $T=0$, and the circles are results of numerical simulations with parameters (excitation power, temperature, cooling rate, lifetimes) chosen at random corresponding to radiative branching ratio $\eta$ ranging between $3 \times 10^{-4}$ and 0.3 . 
We have developed a theoretical analysis of the spectrally resolved PEC signals that includes band filling effects. This model describes well all the major features observed in experiments at low as well as at high initial photoexcited carrier concentrations. We found two limit regimes with different behavior of the $\mathrm{PEC}$ signals depending on whether the semiconductor is degenerate or not. In the nondegenerate regime, which corresponds to the case analyzed in previous studies, an increase in photoinjected carrier density causes an increment of the population at all energies and a superlinear PL response. This leads to completely positive PEC signals and exponential-like decays. In contrast, in the degenerate regime the levels located at the bottom of the conduction band are completely filled with electrons and further injection of carriers increases the population at high energy levels. This leads to sublinear behavior with negative PEC signals at the bottom of the band and superlinear behavior with positive signals at the top of the band.

The simulated PEC spectra and signal decays are in very good agreement with those experimentally measured from a multiple-quantum-well sample. Furthermore, we have shown that fitting of measured decay signals with the predictions of the model allows the measurement of lifetimes which agree with independent PL measurements conducted using a synchroscan streak camera. Other parameters, such as carrier density, are also obtained from the fittings. It should be noted that the theoretical analysis allows for measurements to be conducted in the nondegenerate as well as in the degenerate regime, where lifetime measurements were not possible with the previ- ous understanding of this technique. The latter regime is of significant practical interest because applications such as the semiconductor lasers require the study of materials at high injected carrier densities.

Previous works have established that the PEC signal and the radiative efficiency of the material are related. Our theory shows that there is a relation between the ratio of areas negative over positive in the PEC spectrum and the rate of increase of the radiative efficiency. This relation can be analytically found at $T=0 \mathrm{~K}$ and numerical simulations show that the relation is practically universal.

In conclusion, we have developed a theory that explains the spectral distribution and the different time decay behavior of the PEC signals obtained under low and high photoexcitation conditions. These results expand the utility of this sensitive ultrafast technique as a tool for the study of carrier dynamics in semiconductor materials.

\section{ACKNOWLEDGMENTS}

The authors wish to thank Professor G. Robinson for providing the multiple quantum well samples. We also wish to thank Professor J. Mahan for lending the cryostat and Dr. S. Goodnick for helpful discussions. This work was supported by the Center for Optoelectronic Computer Systems through National Science Foundation Grant No. ECD 9015128 and by the Colorado Advanced Technology Institute Grant No. 1537757.
${ }^{1}$ D. Rosen, A. G. Doukas, Y. Budansky, A. Katz, and R. R. Alfano, Appl. Phys. Lett. 39, 935 (1981).

${ }^{2}$ D. von der Linde, J. Kuhl, and E. Rosengart, J. Lumin. 24/25, 675 (1981).

${ }^{3}$ M. B. Johnson and T.C. McGill, J. Appl. Phys. 63, 2077 (1988).

${ }^{4}$ N. Sawaki, R. A. Höpfel, and E. Gornik, Appl. Phys. Lett. 55, 1996 (1989).

${ }^{5}$ M. K. Jackson, M. B. Johnson, D. H. Chow, and T. C. McGill, Appl. Phys. Lett. 54, 552 (1989).

${ }^{6}$ R. Strobel, R. Eccleston, J. Kuhl, and K. Kohler, Phys. Rev. B 43, 12564 (1991).

${ }^{7}$ E. Okuno, T. Hori, N. Sawaki, I. Akasaki, and R. A. Höpfel, Jpn. J. Appl. Phys. 31, L148 (1992).

${ }^{8}$ R. Christanell and R. A. Höpfel, Superlatt. Microstruct. 5, 193 (1989).
${ }^{9}$ M. Jørgensen and J. M. Hvam, Appl. Phys. Lett. 43, 460 (1983).

${ }^{10}$ H. J. W. Eakin and J. F. Ryan, J. Lumin. 40/41, 553 (1988).

11 J. Shah, T. Damen, B. Deveaud, and D. Block, Appl. Phys. Lett. 50, 1307 (1987).

${ }^{12}$ M. J. Hafich, H. Y. Lee, G. Y. Robinson, D. Li, and N. Otsuka, J. Appl. Phys. 69, 752 (1991).

${ }^{13} \mathrm{C}$. Weisbuch and B. Vinter, Quantum Semiconductor Structures (Academic Press, San Diego, 1991).

14 J. Shah, in Hot Carriers in Semiconductor Nanostructures, edited by J. Shah (Academic Press, San Diego, 1992).

15 J. S. Blakemore, Semiconductor Statistics (Dover Publications, New York, 1987).

${ }^{16}$ P. Vashishta and R. K. Kalia, Phys. Rev. B 25, 6492 (1982). 